\title{
GCU
}

Glasgow Caledonian

University

University for the Common Good

\section{Teaching athletes to understand their attention is teaching them to concentrate}

Oliver, Alex; McCarthy, Paul J.; Burns, Lindsey

Published in:

Journal of Sport Psychology in Action

DOI:

$10.1080 / 21520704.2020 .1838980$

Publication date:

2021

Document Version

Author accepted manuscript

Link to publication in ResearchOnline

Citation for published version (Harvard):

Oliver, A, McCarthy, PJ \& Burns, L 2021, 'Teaching athletes to understand their attention is teaching them to concentrate', Journal of Sport Psychology in Action, vol. 12, no. 3, pp. 196-210.

https://doi.org/10.1080/21520704.2020.1838980

\section{General rights}

Copyright and moral rights for the publications made accessible in the public portal are retained by the authors and/or other copyright owners and it is a condition of accessing publications that users recognise and abide by the legal requirements associated with these rights.

Take down policy

If you believe that this document breaches copyright please view our takedown policy at https://edshare.gcu.ac.uk/id/eprint/5179 for details of how to contact us. 
2

3

4

5

6

$7 \quad{ }^{1}$ Department of Psychology, School of Health and Life Sciences, Glasgow Caledonian 8

9

10

11

12

13

14

15

16

17 This is an accepted manuscript (9/10/20) of an article published by Taylor \& Francis in The

18 Journal of Sport Psychology in Action. Available online at:

19 https://doi.org/10.1080/21520704.2020.1838980.

\author{
Alex Oliver ${ }^{1}$ \\ Paul J. McCarthy ${ }^{1}$ \\ Lindsey Burns ${ }^{1}$
}

University

All correspondence related to this article should be addressed to Alex Oliver by email alex.oliver@gcu.ac.uk or in writing to:

Department of Psychology, School of Health and Life Sciences, Glasgow Caledonian University, Cowcaddens Road, Glasgow, G4 0BA. 
20 Abstract:

21 Concentration, focusing on the most relevant information for further processing, is regarded 22 as a prerequisite for successful sporting performance. Athletes must possess awareness, 23 knowledge, and controllability of their attention to concentrate effectively. One way to 24 develop this awareness, knowledge, and controllability of attention is through the mechanism 25 of meta-attention - thinking about, knowing about, and controlling attention. Meta-attention 26 illuminates the underlying cognitive mechanisms that direct the attentional spotlight. We 27 open with a brief background of attention and outline how concentration can be understood through a metacognitive lens. Next, we present Think Aloud Level 3 as a tool for capturing meta-attentional processes during sport performance. Finally, we discuss the applied implications of adopting a meta-attentional approach to understand concentration.

\section{Introduction}

33 Attention refers to selecting information for further cognitive processing consciously or 34 unconsciously and involves the brain focusing and managing sensory inputs towards 35 information perceived relevant to successfully complete the task at hand while inhibiting other information from further processing (Smith \& Kosslyn, 2007). But attention has a

37 limited capacity that emphasises selecting stimuli most relevant to complete the task (Chun et 38 al., 2011). Therefore, a performer should select relevant information for further processing 39 that facilitates performance, otherwise, performance decrements might occur as the performer 40 becomes distracted by task irrelevant information. Concentration, or directing attention, is 41 therefore widely acknowledged as a pre-requisite for successful sporting performance because our attention is always somewhere. 
44 We best understand how attention works through metaphors. The most prominent metaphor in sport and performance psychology describes attention as a 'spotlight' (Posner, 1980; Moran \& Toner, 2017). The attentional spotlight illuminates stimuli and what has been 'litup' is attended to - or concentrated on. The attentional spotlight can shine outwards towards external stimuli or inwards to internal stimuli. The individual must attempt to illuminate the most relevant information or face becoming distracted. Eye-tracking research (e.g., the 'Quiet Eye’; Vickers, 1996) shows that expert athletes are better at selecting visual (i.e., external) information than their novice counterparts (Moran et al., 2018) because experts know "when, and where to look" (Mann et al., 2007: p.457). The Quiet Eye refers to the final fixation before skill movement and this fixation lasts longer in experts than in novices. The Quiet Eye phenomenon has been shown across a range of motor-skills (Vickers, 2016) and its significance in skill execution has been highlighted in meta-analyses (e.g., Rienhoff et al., 2016). Eye-tracking research, however, can only show us so much, for example, it cannot show us why or when individuals direct their attentional spotlight inwards as it only provides gaze data. Therefore, we need to get behind the 'Quiet Eye' if we are to understand concentration in athletes.

To direct their attentional spotlight, athletes must have knowledge and control of their attention system, something that relies on higher-order processes. First, this means that athletes need to know that they are attending to information that is most facilitative of performance at that time. By having knowledge of their attentional system, they will know the impact of their selected information on their attention. Second, athletes need to be able to implement control routines so that they can then attend to the most relevant information for performance. Therefore, knowledge and control of the attentional spotlight allows an athlete to focus and re-focus their attention during a performance. Without understanding what 
athletes think about their own attention, practitioners cannot offer the most effective interventions to their athletes. This article presents an understanding about how athletes direct their attentional spotlight, outlines a methodological approach that can measure and gain insight into the control of the spotlight and provides considerations for practice. First, we explain metacognition and meta-attention and its significance to understand concentration. Next, we explain how 'Think Aloud' (Ericsson \& Simon, 1993) can explore the metaattentional processes of athletes. Finally, we consider applied implications of a metaattentional approach to understand concentration.

\section{Metacognition, meta-attention and its significance for understanding concentration.}

The study of metacognition helps us to understand how athletes think about and control their cognitive processes in performance. Metacognition is an individual's insight and control over their cognitive processes (Flavell, 1979), and it covers each cognition (e.g., meta-emotion, meta-memory, and meta-attention). This means that each cognition we experience has an associated meta-level process. Metacognition is commonly simplified to thinking about thinking or "knowing about knowing” (Van Overschelde, 2008, p. 47), however, metacognition is broader than its frequent simplification and can be best understood as a tripartite construct comprising: knowledge (e.g., of cognitive strategies and when to use them), control, and monitoring (Flavell, 1979; Flavell, 1987; Halpern, 2014; Tarricone, 2011). Therefore, metacognition is considered as fundamental for task performance and effective self-regulation because it allows controlling thoughts and actions to meet task demands (Dinsmore et al., 2008). Metacognition can range from a subconscious monitoring of thoughts to a more detailed reflection of the self, therefore it does not always require awareness or control (MacIntyre et al., 2014; Shea et al., 2014) and may take place in cognitive system 1 (see: Stanovich, 2011). System 2 is involved in metacognitive processes 
where automatic processes would not be sufficient for processing the information faced, such as during more complicated tasks that require reflection or decision-making (MacIntyre et al., 2014). System 1 is automatic and outside of consciousness and, system 2 is conscious and is engaged when an individual faces a more complicated task that relies on an effortful search and control of cognitions.

The origins of metacognition and metacognitive research relate to education, with many conceptualisations about metacognitive learning. For example, Flavell (1979) considered metacognitive knowledge crucial for students because it enabled self-directed learning. Students proficient in metacognitive knowledge possess awareness of their knowledge, what they need to know and the study techniques that facilitate further knowledge, thus are more effective learners than their peers who do not possess this proficiency. Much of the research has built on Flavell's (1979) work and remained within an education and learning setting (Hacker et al., 2008). Because intellectual skills and perceptual-motor skills rely on similar mechanisms, metacognition may account for perceptual-motor skills just as they do with intellectual skills (Augustyn \& Rosenbaum, 2005; MacIntyre et al., 2014). Therefore, metacognition is a strong theoretical concept from mainstream psychology that applies in sport psychology. Taking this understanding forward, proficiency in metacognition can be linked to skill development because the most successful athletes are likely to have knowledge of their current skillset (i.e., perceptual-motor skills, perceptual-cognitive, technical, and tactical), and the skills that require further development to achieve success (MacIntyre et al., 2014; Moran et al., 2019). Further, by using 'skills' to describe metacognition it implies trainability of metacognitive proficiency, that is, metacognition is not necessarily a fixed trait-like characteristic so an increase in an athlete's domain-specific knowledge will increase the athlete's ability to be use metacognitive strategies in performance. 
120 Meta-attention, a component of metacognition, can provide insights into the controllability of

121 attention and concentration because it is thinking about, knowing about and, controlling 122 attention. Meta-attention holds great promise for better understanding concentration among 123 athletes because the study of meta-attention illuminates the underlying cognitive mechanisms 124 that direct the attentional spotlight (Moran, 1996). As we outlined earlier, an athlete's focus 125 of attention can have a significant influence on performance outcomes because an athlete must select the most relevant stimuli or become distracted.

128 The original understanding of meta-attention is rooted in education, with research showing 129 that during a reading task younger children perceive their attention to be controlled by external variables (e.g., distractions such as noise and reward), whereas older children 131 possess awareness of internal variables (e.g., interest) that can be controlled to direct 132 attention to maintain task relevant focus. In a notable study, Loper and Hallahan (1982) 133 measured meta-attention in school-age children (Kindergarten; Grade 2; Grade 5; and Grade 134 7) by using variables relevant to attention: interest, reward and noise distraction, in a forced choice measure. Each variable had a positive and a negative scenario that were either aids or deterrents to attention (e.g., "imagine a child who is very interested in what he is doing";

137 "imagine a child who is not very interested in what he is doing”). Cards were then paired to 138 establish forced choice items and children were asked, "which of these two children will 139 better pay attention?” Scoring was then calculated by the number of times a high value card 140 was chosen and the number of times a low value card was not (e.g., the number of times high 141 interest was chosen and the number of times low interest was not). Scoring was performed 142 for three measures: 'interest', 'reward', and 'quiet'. Each of the scenarios were paired against 143 each other, to present a variety of situations, and each card was presented five times within 
144 twelve forced choices. Reading achievement was measured by using the validated measure

145 Wide Range Achievement Test (1965); a word recognition task. This test, however, was not

146 given to the youngest age group. Loper and Hallahan's (1982) findings supported their

147 hypotheses, showing that older children valued interest highest whereas younger children

148 valued reward and noise distraction. Post-hoc evaluations showed there to be significant age-

149 group differences on the interest variable. The research indicates that older children are more

150 aware of the variables required for attention and that they are able to perceive distractors too.

151 Therefore, older children are in a better position to control arising distractors, avoiding

152 disruptions to task performance (Loper \& Hallahan, 1982; Miller \& Bigi, 1979). Older

153 children possess awareness of their attention, therefore, know techniques that facilitate

154 concentration and those that do not. Loper and Hallahan (1982) also used Karniol and Ross

155 (1979) to help explain the differences between older and younger children's attention in the

156 reading task, outlining that it is the maturity of the reader that creates a disposition to

157 discount the value of extrinsic rewards because this may disrupt internal, intrinsic,

158 enjoyment. Therefore, changes in meta-attention are related to cognitive development.

159 Applied to sport, this means that performance related cognitions are likely to become more 160 developed with greater experience and greater knowledge. For example, an awareness of 161 standards and expectations and of cognitions that are conducive of performance. That is, 162 planning, monitoring and control of cognitions and behaviours and ensuring these are 163 consistent with the expected level. Loper and Hallahan (1982) also showed a small, but 164 significant, positive correlation between interest and reading achievement. Thus, 165 understanding meta-attention here can be related to the understanding that optimal concentration requires focusing solely on actions under one’s control (Moran, 2011). The

167 focus of attention may be 'lost' without an environmental distractor (i.e., external) present, 168 instead a wandering mind can direct attention towards task irrelevant thoughts (i.e., internal) 
that result in lost concentration (Moran, 1996; Moran, 2011). The mechanism that realises

170 sufficient attention is not directed to task-relevant information is a monitoring system that

171 facilitates a re-focus to the task can and be identified as meta-attention and mapped onto the

172 metacognitive framework. The accuracy and effectiveness of the meta-attentional mechanism

173 is likely linked to the efficiency of an individual's cognitive processing (Moran, 1996).

174 Because of a predicted greater metacognitive proficiency among higher-skilled athletes

175 (MacIntyre et al., 2014), it is assumed that such athletes have a greater capacity to focus their

176 attention, and are more aware of a wandering mind and are therefore able to implement

177 suitable strategies to re-focus on task relevant cues allowing for successful control of 178 attention.

179

180 Although meta-attention has only recently been examined in a sport context, some insight can

181 be gained by looking at metacognitive research that sought to examine the role of 182 metacognition in attentional focus. For example, Brick et al. (2015) highlighted the important 183 role of metacognition for attentional focus in an endurance sport setting by creating a 184 framework relevant to endurance activity, driven by interview data with elite endurance 185 runners. Their data revealed that athletes monitor and control cognitions during running to optimise performance; specifically, athletes engaged in planning attentional strategies, monitoring task performance and, control of cognition during running. Results also indicated

188 that athletes engaged in a review, an evaluation and refined their cognitions after running;

189 behaviour that can be mapped to self-regulatory behaviours (e.g., Zimmerman, 2006).

191 Nietfeld (2003) indicated that the effectiveness of metacognitive strategy in performance is underpinned by the ability of the athlete. In a one-mile treadmill running task, Nietfeld 193 (2003) reported a positive relationship between metacognitive strategy knowledge and the 
ability of runners to monitor their performance. The findings here indicated that skilled performers monitored largely internally and used domain-specific strategies driven by their experience. Internal monitoring links to the understanding of meta-attention from an educational context that searches for internal variables deemed to be important for performance occurred, whereas strategies, can be linked to conscious control of attention.

199 Monitoring and information management strategies (i.e., control) were runners most frequent reported attentional focus. Nietfeld's (2003) study also showed a negative correlation between an external focus and metacognition, showing that metacognition is an internal process. A positive correlation was reported between strategic knowledge and pace monitoring; therefore, athletes should require awareness of suitable strategies and tactics to most effectively monitor their performance. Overall, the findings in Nietfeld's study show that skilled athletes are highly focused and strategic, processes that are seen to be optimal for performance. It is suggested that their domain-specific strategies are developed through their experience. Similar research using recreational runners by Brick et al. (2018) showed that novice runners did not report a repertoire of metacognitive strategies, pacing and tactics were reported, however, planning was not. In addition, post-race evaluations were not conducted independently, rather were done with the help of more experienced runners. The results from

211 this study showed that novice runners were less strategic (i.e., planning) and were less likely 212 to plan or evaluate attentional strategies during running (i.e., monitoring). Brick et al. (2018) 213 outlined that metacognitive ability and cognitive strategies were developed through running 214 experience and this helped, runners shifting their attentional focus. Through newly gained metacognitive abilities, runners were aware of sensory cues as an information source relevant

216 for their pace decision-making. By using MacIntyre et al. (2014) and Bless et al. (2009) 217 conceptualisation of metacognition, experience could be explained by expert and higher218 skilled athletes' possessing greater declarative knowledge (i.e., 'knowing that'; Sternberg \& 
219 Sternberg, 2016). Experts are then able to judge whether information is relevant, and take it

220 forward for greater processing, or if it is not relevant, to discard that information. Therefore,

221 practitioners should focus on developing declarative knowledge with their athletes because

222 this will teach them to monitor for relevant cues across situations. Thus, with declarative

223 knowledge athletes are more able to direct their attentional spotlight. Brick et al.’s (2018)

224 findings add support to the previous research that indicates the important role of task-specific metacognitions that best facilitate regulation of cognitions such as attentional focus. To date,

226 the findings from studies that adopt a metacognitive framework to better understand 227 attentional processes in performance are bound by the context specificity of endurance sports.

229 To investigate meta-attention further Oliver et al., (2020a) used a grounded theory to understand meta-attention among competitive-elite and successful-elite golfers. The resultant theory showed that meta-attention operated as a bottom-up process with attentional resources evaluated by golfers before using attentional control strategies; selecting an external visual target, implementing a pre-shot routine and a consistent post-shot routine. In this case, attentional resources can be viewed as internal variables and include, but are not limited to: experience, training and confidence. Golfers experienced optimal attentional outcomes after positively perceiving their attentional resources and implemented consistent attentional control strategies; however, should a resource be perceived missing when required or performers did not implement a control strategy, they experienced sub-optimal attentional outcomes and faced internal distraction. The golfers' understanding of their own attentional system drove meta-attentional processes and selection of desired attentional processes.

241 Evaluating attentional resources and implementing a control strategy uncovered in Oliver et 242 al.’s (2020a) study shows that golfers plan, monitor and control their attention regulation 243 during performance. 
245 Building on these findings, Oliver et al. (2020b) explored meta-attention in a live setting using concurrent data collection of meta-attentional processes during golf performance. By using concurrent data collection, the researchers developed their previous study by exploring how golfers engage in attentional thought and control during performance, capturing thoughts and control strategies as they unfold. Golfers drew on different metacognitive resources (i.e., internal) at different times, however, starting control strategies were far more prevalent than verbalisations of attention-based resource reflections. These findings suggest that monitoring of internal resources is generally an unconscious process, however, during more challenging times it may require an athlete to make a conscious search for a specific attentional resource. For example, when golfers faced a tough shot or a recovery shot, they reflected on practices and coaching they had received verbalising that they intended to draw on these experiences to help them execute the shot they faced. Identifying unconscious monitoring allows for theoretical integration with Stanovich's (2011) depiction of two cognitive systems. Golfers engaged in a post-shot routine and 'switched-off', shifting their attention to a task-irrelevant focus, between shots. This shifting of attention supports Davies et al. (2014) findings about a golfer’s attentional processes between shots and substantiates Tiger Woods’ (2001) anecdotal example that an evaluation should take place before an attentional shift towards the next shot begins. The post-shot thoughts shared by the golfers also extend Kirschenbaum's 4-F model (1997), expanding from a negative shot outcome to one for all shot outcomes.

Further meta-attentional research by Oliver et al. (under review) showed that higher skilled golfers (Mean Handicap= 5.7) reported significantly more verbalisations per shot than lower skilled golfers (Mean Handicap= 23), suggesting greater meta-attentional knowledge (i.e., more awareness of where their attentional beam was located). The results of Oliver et al.'s 
269 (under review) study lend some support to MacIntyre et al.’s (2014) suggestion that expertise

270 links with metacognition because higher skilled golfers verbalised more thoughts about their

271 attentional cognitions. We are also cautious with this assertion because Oliver et al.'s (under

272 review) study used two levels of club golfers, thus the extent to which the higher-skilled

273 golfers possessed expertise could be questioned. Nevertheless, Oliver et al.'s (under review)

274 study shone a light on golfers' attentional spotlight and concentration. Thought sequencing

275 showed that higher skilled golfers verbalisations showed two lots of information gathering,

276 suggesting that they spent longer gathering information on their environment, thus, could be

277 seen to develop more informed plans to assist with successful skill execution. Sequential

278 analysis was performed and it indicated likely thought pairings, revealing the most likely

279 preceding thoughts, i.e., thoughts that occurred at a probability greater than chance. Thought

280 pairings that occurred at a greater probability than chance suggest a deliberate decision to

281 divert the attentional spotlight to information, either internally or externally, that the golfer

282 judged most relevant.

284 Capturing Meta-Attentional Thoughts: Using Think Aloud.

285 Think Aloud (TA) is a research method outlined by Ericsson and Simon (1993) to overcome

286 retrieval issues related to cognitive processes and experiences which are not an observable

287 behaviour. Therefore, TA is a tool that can help us get behind the 'Quiet Eye' and capture

288 conscious-level metacognitive processes involved in directing the attentional spotlight. We

289 do, however, acknowledge that TA can only provide insight into processes occurring in 290 cognitive system 2. Nevertheless, system 2 is where we see effortful planning, monitoring

291 and control of physiological and psychological processes in a sport performance, something

292 that previous research indicated expert performers do. Processes that are verbal are ideal for 
293 TA research, thus thinking about attention and vocalising strategies deployed to control attention (i.e., meta-attention) is a prime candidate for research and applied practice.

295

There are three levels of TA (Ericsson \& Simon, 1993), Level 1 is an effortless

297 communication of inner speech and Level 2 refers to verbalising an internal representation 298 that is not originally in verbal code (e.g., visual stimuli). Level 3 is at a much more detailed level than Level 1 and 2 because it requests individuals to explain their thoughts, ideas and motives. Therefore, Level 3 is closest to accessing the meta- level because it requires individuals to think about their thinking (Oliver et al., 2020b). For example, a golfer 302 explaining why they are playing a certain shot and the factors they consider making this 303 decision. To achieve Level 3 verbalisation, there is a requirement that the participant links between information and previously attended to thoughts. This means that Level 3 TA includes retrieval from Long Term Memory (i.e., where the explanation is housed). Because individuals consciously attend to additional information, that would otherwise be automatic,

307 there is some concern that this focus could disrupt thought processes and performance (e.g.,

308 lead to reinvestment: Masters, 1992; Masters \& Maxwell, 2008), however, research by 309 Whitehead and colleagues (2015) allayed these fears and showed that within Level 3 TA golfers did not have their performance adversely affected by engaging in TA. Therefore, TA

311 is a suitable tool to capture, measure and understand the cognitive processes experienced by

312 athletes during performance with no detriment to performance. TA is also a useful tool for

313 practitioners to better understand the cognitive processes that their athletes experience during 314 performance.

316 To further highlight TA's suitability for collecting data on cognitive processes experienced 317 by athletes during performance, is the growing popularity of the successful application of TA 
across sport, including but not limited to: golf (Calmeiro \& Tenenbaum, 2011; Nicholls \&

319 Polman, 2008; Oliver et al., 2020b; Oliver et al, under review; Whitehead et al., 2015;

320 Whitehead et al., 2016), trapshooting (Calmeiro et al., 2010), tennis (McPherson \& Kernodle,

321 2007), snooker (Walsh et al., 2018) and cycling (Whitehead et al., 2019). TA allows us to

322 measure cognitive processes during a performance which can be mapped, and taken forward

323 to inform interventions. Athletes should be briefed on TA and undertake practice trials that

324 act as a warm-up and familiarisation to using TA (Eccles \& Arsal, 2017). Previous research

325 suggests that those completing TA are handed and narrated Level 3 TA instructions outlining

326 what is asked for them. The instructions given to participants should be sport specific for

327 example golfers are instructed to verbalise where they directed their attention before a chip

328 shot and why attention was to be directed there (Oliver et al., 2020b). Athletes should be

329 given the opportunity to ask questions they have on Think Aloud. Practice trials can then

330 provide familiarity with verbalising aloud (Eccles, 2012). Recent research outlines that

331 practice trials may be context specific (Birch \& Whitehead, 2020), however, to date much of

332 the research has used non-task specific tasks such as calculating the number of dots on a

333 page. The importance in familiarity and warm-up tasks shows the practitioner that athletes

334 understand what is being asked of them when thinking aloud. By explaining their thoughts

335 and actions, Level 3 TA provides an approximation to metacognitive processes because it

336 required the golfers to think about their thinking (Oliver et al., 2020b).

338 Capturing meta-attentional verbalisations therefore allows practitioners to map the direction

339 of the attentional beam, for example, when and why athletes divert it inwards at certain points

340 during their performance. TA may also be of direct benefit to performers too as verbal reports

341 can provide athletes insights into their own thought patterns, for example, it allows them to

342 identify where thoughts have been helpful and unhelpful, this can then be used as part of their 
343 own learning, development and may benefit the development of metacognition. Therefore,

344 TA is posited as a suitable tool for understanding attentional control because it provides

345 insights to the higher-level executive (i.e., meta- level) functions involved in orienting 346 attention, something that can be used by athletes and practitioners.

\section{Teaching athletes how to concentrate}

349 Using meta-attention to understand concentration has several implications for athletes, coaches and sport psychologists. First, meta-attention shows that understanding the attentional system drives meta-attentional processes and selecting desired attentional

352 processes. Those who are most proficient in their meta-attention can accurately direct their

353 spotlight to the most relevant stimuli at the required time. By understanding their internal and external attentional stimuli athletes can more accurately direct their attentional spotlight and are less likely to succumb to distraction (i.e., illuminating task irrelevant stimuli). Specifically, our current understanding of meta-attention (e.g., Oliver et al., 2020a) shows an

357 evaluation of attentional resources takes place; should an individual experience cognitive anxiety related to their attentional resources their perception of their resource can become an internal distraction. Applying this process to practice, concentration could be enhanced by teaching performers to avoid using binary evaluations of their attentional resources. Oliver et al.’s (2020a) research shows golfers evaluate their attentional resources through a positivenegative lens, they either have the attentional resource required or they do not. Using a binary evaluation on attentional resources may be the source of the problem, rather than the missing resource itself, because it is the negative perception that becomes the source of distraction. Therefore, athletes should avoid using a positive-negative evaluation of their attentional resources. To help performers avoid binary evaluations of their attentional resources, it may

367 be worthwhile for golfers to undertake mindfulness training (e.g., Birrer et al., 2012; Birrer \& 
368 Röthlin, 2017) because being mindful takes away the binary evaluation that occurs in the

369

370

371

372

373

374

375

376

377 378 level.

379

380

381

382

383

384

385

386

387

388

389

390

391

392 monitoring stage of meta-attention. We also believe that meta-attention and mindfulness are conceptually linked because proficiency in each promote a focus on the present moment. If athletes are mindful they can bring their attention to the present moment, that is, they can direct their attentional spotlight to the most important information but to do so they need to know where their spotlight is shining in the first place (i.e., having meta-attentional awareness). Mindfulness-based interventions may also benefit golfers who do not have a full repertoire of the attentional resources outlined by Oliver et al.'s (2020) model because a mindful-based approach could offset negative perceptions of 'missing' resources. Practitioners could use a mindfulness-based intervention with athletes regardless of their skill

Another practical implication from meta-attentional research relates to controlling attentional shifts. Pre-shot routines for switching on attention is well established for self-paced sports (e.g., Cotterill et al., 2010) and is widely applied as an intervention in practical sport psychology settings. But there has been less attention in the literature that seeks to understand how golfers use the time between shots. The ways golfers make use of this time provides details on effective attention regulation, and allows for attention control and spotlight shifts to be mapped. Oliver et al.’s (2020a; 2020b; under review) findings showed that golfers took on a task irrelevant focus during periods between skill executions as a final stage in their post-shot routine. These findings offer a recommendation that those working with golfers instruct them to implement a post-shot routine to allow for feedback to take place. Then, golfers should initiate a switch-off period between shots. The downtime between shots is taken up by a task irrelevant focus. When coaches and sport psychologists are developing an attention training package post-shot routine training and switch-off should be delivered 
393 alongside a pre-shot routine. Providing training on pre-shot and post-shot routines within the same intervention package will give golfers' greater control of their attention shifts that will occur in a round of golf. This pairing can also increase athletes' awareness and knowledge of their attention. The practical suggestion of the benefits that a post-shot routine and between-

397 shot switch-off bring indicated by Oliver et al.’s research (2020a; 2020b; under review) is 398 consistent with previous research in golf (Davies et al., 2014; Davies et al., 2017; 399 Kirschenbaum, 1997). A further benefit of training post-shot routines and disassociation is that golfers preserve attentional resources required for attentional control throughout a round of golf. A post-shot routine may resemble an immediate outcome reaction (good or bad), a

402 technical evaluation and a task irrelevant focus. Anecdotal evidence suggests that golfers 403 "switch off" - or disassociate - between shots because it is unfeasible to be focused throughout an entire round of golf. Applied sport psychologists may wish to apply these principles to athletes from other intermittent and target sports so that their athletes optimise their concentration, and gain greater control of their attentional spotlight.

To conclude, this article has shown that meta-attention can explain distractibility in sports performers and describe ways in which an athlete focuses and re-focuses attention. Concentration depends on positive evaluations of attentional resources when they are

411 required and implementing a suitable control strategy pre- and post- skill execution because 412 these strategies allow performers to direct their attentional spotlight. This is a process that is 413 underpinned by an athlete’s meta-attentional proficiency. Because attention is always shining somewhere and metacognitive processes are on-going, TA can be used to improve spotlight 415 accuracy because it makes performers more aware of their attentional processes. If 416 performers are aware of where their spotlight is shining and where it should be shining they 417 can direct it if it is misplaced. Therefore, TA would increase accuracy of their attentional 
418 spotlight. We suggest that practitioners explore their athletes' understanding and knowledge

419 of attention in live performance settings, and create a bespoke training programme to 420 optimise concentration. This is achieved by showing athletes where their attention is, in turn

421 handing athletes more awareness, greater control of their attentional spotlight; teaching them 422 how to concentrate.

423

424 Word Count: 4,848 (inc. abstract). 
426 Augustyn, J. S., \& Rosenbaum, D. A. (2005). Metacognitive control of action: Preparation

427

428

429 for aiming reflects knowledge of fitt's law. Psychonomic Bulletin \& Review, 12(5), 911-916. https://doi.org/10.3758/BF03196785

Birch, P. D., \& Whitehead, A. E. (2020). Investigating the Comparative Suitability of Traditional and Task-Specific Think Aloud Training. Perceptual and Motor Skills, 127(1), 202-224. https://doi.org/10.1177/0031512519882274

Birrer, D., \& Röthlin, P. (2017). Riding the third wave: CBT and mindfulness-based interventions in sport psychology. In S. J. Zizzi \& M. B. Andersen (Eds.), Being mindful in sport and exercise psychology (pp. 101-122). FiT.

Birrer, D., Röthlin, P., \& Morgan, G. (2012). Mindfulness to enhance athletic performance: Theoretical considerations and possible impact mechanisms. Mindfulness, 3(3), 235246. https://doi.org/10.1007/s12671-012-0109-2

Bless H., Keller J., Igou E. R. (2009). Metacognition. In J. Förster \& F. J. Strack (Eds.). Social Cognition, the Basis of Human Interaction (pp. 157-178). Psychology Press.

Brick, N. E., Campbell, M. J., Sheehan, R. B., Fitzpatrick, B. L., \& MacIntyre, T. E. (2018). Metacognitive processes and attentional focus in recreational endurance runners. International Journal of Sport and Exercise Psychology, 16, 1-18. https://doi.org/10.1080/1612197X.2018.1519841

Brick, N. E., MacIntyre, T. E., \& Campbell, M. J. (2015). Metacognitive processes in the self-regulation of performance in elite endurance runners. Psychology of Sport and Exercise, 19, 1-9. https://doi.org/10.1016/j.psychsport.2015.02.003

Calmeiro, L., \& Tenenbaum, G. (2011). Concurrent verbal protocol analysis in sport: Illustration of thought processes during a golf-putting task. Journal of Clinical Sport Psychology, 5(3), 223-236. https://doi.org/10.1123/jcsp.5.3.223 
450 Calmeiro, L., Tenenbaum, G., \& Eccles, D. (2010). Event-sequence analysis of appraisals

451

452

453

454

455

456

457

458

459

460

461

462

463

464

465

466

467

468

469

470

471

472

473

474 and coping during trapshooting performance. Journal of Applied Sport Psychology, 22(4), 392-407. https://doi.org/10.1080/10413200.2010.495325

Chun, M. M., Golomb, J. D., \& Turk-Browne, N. B. (2011). A taxonomy of external and internal attention. The Annual Review of Psychology, 62, 73-101. https://doi.org/10.1146/annurev.psych.093008.100427

Cotterill, S. T., Sanders, R., \& Collins, D. (2010). Developing effective pre- performance routines in golf: Why don't we ask the golfer? Journal of Applied Sport Psychology, 22(1), 51-64. https://doi.org/10.1080/10413200903403216

Davies, T., Collins, D., \& Cruickshank, A. (2014). So what do we do with the rest of the day? going beyond the pre-shot routine in professional golf. International Journal of Golf Science, 2, 163-175. https://doi.org/10.1123/ijgs.2014-0008

Davies, T., Collins, D., \& Cruickshank, A. (2017). This is what we do with the rest of the day! exploring macro and meso levels of elite golf performance. The Sport Psychologist, 31(2), 117-128. https://doi.org/10.1123/tsp.2016-0049

Dinsmore, D. L., Alexander, P. A., \& Loughlin, S. M. (2008). Focusing the conceptual lens on metacognition, self-regulation, and self-regulated learning. Educational Psychology Review, 20(4), 391-409. https://doi.org/10.1007/s10648-008-9083-6

Eccles, D. W. (2012). Verbal reports of cognitive processes. In G. Tenenbaum, R. C. Eklund \& A. Kamata (Eds.), Measurement in sport and exercise psychology (pp. 103-117). Human Kinetics.

Eccles, D. W., \& Arsal, G. (2017). The think aloud method: What is it and how do I use it? Qualitative Research in Sport, Exercise and Health, 9(4), 514-531. https://doi.org/10.1080/2159676X.2017.1331501

Ericsson, K. A., \& Simon, H. A. (1993). Protocol analysis. The MIT Press. 
475 Flavell, J. H. (1979). Metacognition and cognitive monitoring: A new area of Cognitive476 Developmental inquiry. American Psychologist, 34(10), 906-911. https://doi.org/10.1037/0003-066X.34.10.906

478 479 480

Hacker, D. J., Bol, L., \& Keener, M. C. (2008). Metacognition in education: A focus on calibration. In J. Dunlosky \& R. A. Bjork (Eds.), Handbook of metamemory and memory (p. 429-455). Psychology Press.

Halpern, D. F. (2014). Thought and knowledge: An introduction to critical thinking (5th ed.). Psychology Press.

Jastak, J. F., \& Jastak, J. F. (1965). The Wide Range Achievement Test. Guidance Association.

Karniol, R., \& Ross, M. (1979). Children's use of a causal attribution schema and the inference of manipulative intentions. Child Development, 463-468. https://doi.org/10.2307/1129424

Kirschenbaum, D. S. (1997). Mind matters: Seven steps to smarter sport performance. Cooper.

Loper, A. B., \& Hallahan, D. P. (1982). Meta-attention: The development of awareness of the attentional process. The Journal of General Psychology, 106(1), 27-33. https://doi.org/10.1080/00221309.1982.9710970

MacIntyre, T. E., Igou, E. R., Campbell, M. J., Moran, A. P., \& Matthews, J. (2014). Metacognition and action: A new pathway to understanding social and cognitive aspects of expertise in sport. Frontiers in Psychology, 5, 1-12. https://doi.org/10.3389/fpsyg.2014.01155

Mann, D. T., Williams, A. M., Ward, P., \& Janelle, C. M. (2007). Perceptual-cognitive expertise in sport: A meta-analysis. Journal of Sport and Exercise Psychology, 29(4), 457-478. https://doi.org/10.1123/jsep.29.4.457 
500 Masters, R. S. W. (1992). Knowledge, knerves and know-how: The role of explicit versus

501

502

503

504

505

506

507

508

509

510

511

512

513

514

515

516

517

518

519

520

521

522

523

524 implicit knowledge in the breakdown of a complex motor skill under pressure. British Journal of Psychology, 83(3), 343-358. https://doi.org/10.1111/j.20448295.1992.tb02446.x

Masters, R. S. W., \& Maxwell, J. (2008). The theory of reinvestment. International Review of Sport and Exercise Psychology, 1(2), 160-183. https://doi.org/10.1080/17509840802287218

McPherson, S. L., \& Kernodle, M. (2007). Mapping two new points on the tennis expertise continuum: Tactical skills of adult advanced beginners and entry-level professionals during competition. Journal of Sports Sciences, 25(8), 945-959. https://doi.org/10.1080/02640410600908035

Miller, P. H., \& Bigi, L. (1979). The development of children's understanding of attention. Merrill-Palmer Quarterly, 25, 235-250.

Moran, A. P. (1996). The psychology of concentration in sport performers. Psychology Press. Moran, A. P., \& Toner, J. (2017). A Critical Introduction to Sport Psychology: A Critical Introduction. Taylor \& Francis.

Moran, A. P., Campbell, M. J., \& Ranieri, D. (2018). Implications of eye tracking technology for applied sport psychology. Journal of Sport Psychology in Action, 9(4), 249-259. https://doi.org/10.1080/21520704.2018.1511660

Moran, A. P., Campbell, M. J., \& Toner, J. (2019). Exploring the cognitive mechanisms of expertise in sport: Progress and prospects. Psychology of Sport and Exercise, 42, 815. https://doi.org/10.1016/j.psychsport.2018.12.019

Nicholls, A. R., \& Polman, R. C. J. (2008). Think aloud: Acute stress and coping strategies during golf performances. Anxiety, Stress \& Coping, 21(3), 283-294. https://doi.org/10.1080/10615800701609207 
525 Nietfeld, J. L. (2003). An examination of metacognitive strategy use and monitoring skills by

526

527

528

529

530

531

532

533

534

535

536

537

538

539

540

541

542

543

544 Smith, E. E., \& Kosslyn, S. M. (2007). Cognitive psychology: Mind and brain. Pearson.

545 Stanovich, K. E. (2011). Rationality and the reflective mind. Oxford: Oxford University

546

547 Sternberg, R. J., \& Sternberg, K. (2016). Cognitive psychology. Nelson Education. 
548 Van Overschelde, J. P. (2008). Metacognition: Knowing about knowing. In J. Dunlosky \& R.

549

550

551

552

553

554

555

556

557

558

559

560

561

562

563

564

565

566

567

568

569

570

571 Woods, T. (2001). How I play golf. Warner Books. Press. https://doi.org/10.1037/0096-1523.22.2.342 https://doi.org/10.1016/j.psychsport.2018.03.003 Exercise Psychology, 17(3), 266-274. https://doi.org/10.1080/1612197X.2017.1292302 situations. Frontiers in Psychology, 6(1974), 1-12. https://doi.org/10.3389/fpsyg.2015.01974

A. Bjork (Eds.), Handbook of metamemory and memory (p. 47-71). Psychology

Vickers, J. N. (1996). Visual control when aiming at a far target. Journal of Experimental Psychology: Human Perception and Performance, 22(2), 342- 354.

Vickers, J. N. (2016). The quiet eye: Origins, controversies, and future directions. Kinesiology Review, 5(2), 119-128. https://doi.org/10.1123/kr.2016-0005

Welsh, J. C., Dewhurst, S. A., \& Perry, J. L. (2018). Thinking Aloud: An exploration of cognitions in professional snooker. Psychology of Sport and Exercise, 36, 197-208.

Whitehead, A. E., Jones, H. S., Williams, E. L., Dowling, C., Morley, D., Taylor, J. A., \& Polman, R. C. (2019). Changes in cognition over a $16.1 \mathrm{~km}$ cycling time trial using think aloud protocol: Preliminary evidence. International Journal of Sport and

Whitehead, A. E., Taylor, J. A., \& Polman, R. C. J. (2015). Examination of the suitability of collecting in event cognitive processes using think aloud protocol in golf. Frontiers in Psychology, 6(1083), 1-12. https://doi.org/10.3389/fpsyg.2015.01083

Whitehead, A. E., Taylor, J. A., \& Polman, R. C. J. (2016). Evidence for skill level differences in the thought processes of golfers during high and low pressure 
572 Zimmerman, B. (2006). Development and Adaptation of Expertise: The Role of Self-

573 Regulatory Processes and Beliefs. In K. Ericsson, N. Charness, P. Feltovich, \& R.

574 Hoffman (Eds.), The Cambridge Handbook of Expertise and Expert Performance (pp.

575 705-722). Cambridge University Press.

576 http://doi.org/10.1017/CBO9780511816796.039 